\title{
Mass-suppressed effects in heavy quark diffusion
}

\section{A. Bouttefeux and M. Laine}

AEC, Institute for Theoretical Physics, University of Bern, Sidlerstrasse 5, CH-3012 Bern, Switzerland

E-mail: bouttefeux@itp.unibe.ch, laine@itp.unibe.ch

ABSTRACT: Many lattice studies of heavy quark diffusion originate from a colour-electric correlator, obtained as a leading term after an expansion in the inverse of the heavy-quark mass. In view of the fact that the charm quark is not particularly heavy, we consider subleading terms in the expansion. Working out correlators up to $\mathcal{O}\left(1 / M^{2}\right)$, we argue that the leading corrections are suppressed by $\mathcal{O}(T / M)$, and one of them can be extracted from a colour-magnetic correlator. The corresponding transport coefficient is non-perturbative already at leading order in the weak-coupling expansion, and therefore requires a nonperturbative determination.

KeYwords: Thermal Field Theory, Heavy Quark Physics, Lattice QCD, Quark-Gluon Plasma

ARXIV EPRINT: 2010.07316 


\section{Contents}

1 Introduction 1

2 Classical picture $\quad 3$

3 Formal derivation of the force-force correlator 5

3.1 Action up to $\mathcal{O}\left(1 / M^{3}\right)$

3.2 Lorentz force up to $\mathcal{O}\left(1 / M^{2}\right) \quad 6$

3.3 Force-force correlator up to $\mathcal{O}\left(1 / M^{2}\right) \quad 7$

3.4 Physical effects up to $\mathcal{O}(T / M) \quad 9$

4 Perturbative evaluation $\quad 11$

4.1 Temporal correlator 11

$\begin{array}{ll}4.2 \text { Transport coefficient } & 12\end{array}$

$\begin{array}{lll}\text { 4.2.1 Setup } & 12\end{array}$

$\begin{array}{lll}\text { 4.2.2 QCD contributions } & 13\end{array}$

4.2.3 Hard Thermal Loop resummation 16

$\begin{array}{lll}4.2 .4 & \text { Summary } & 16\end{array}$

$\begin{array}{lll}5 & \text { Conclusions and outlook } & 17\end{array}$

\section{Introduction}

The properties of heavy quarks, of mass $M$, inserted into a plasma, at a temperature $T$, can be characterized by a number of dispersive or mass terms $\left(F(\mathbf{v}) \simeq M_{\text {rest }}+M_{\text {kin }} \mathbf{v}^{2} / 2+\ldots\right)$ and absorptive or rate coefficients (diffusion constant, kinetic and chemical equilibration rates). In the setting of a heavy ion collision experiment, the hierarchy $T \ll M$ is not necessarily drastic, particularly for charm quarks. If we determine physical quantities as a series in $T / M$, it may then be asked how large such corrections are, and whether they could help for their part to explain the empirical observation that heavy quarks, such as those identified as $D$ mesons after hadronization, appear to interact efficiently with a hot QCD medium [1].

In the case of dispersive corrections, the nature of the series in $T / M$ is well understood at low orders of perturbation theory. Computing thermal effects in unresummed perturbation theory leads to a mass correction of relative magnitude $\mathcal{O}\left(\alpha_{\mathrm{s}} T^{2} / M^{2}\right)$ [2], however taking into account plasma effects, particularly Debye screening, shows that the dominant correction is only suppressed by $\mathcal{O}\left(\alpha_{\mathrm{s}}^{3 / 2} T / M\right)$. For $M_{\text {rest }}$ this is known as the Salpeter correction (cf., e.g., ref. [3]), and a similar effect also exists for $M_{\text {kin }}$ [4]. The purpose of the present investigation is to study the nature of the series for the case of rate coefficients. 
The physics of heavy quark diffusion and kinetic equilibration is closely related to that of Brownian motion, described by the Langevin equation. In the non-relativistic limit, this physics is described by three quantities: the diffusion coefficient, $D$; the momentum diffusion coefficient, $\kappa$; and the drag coefficient, $\eta$. As already pointed out by Einstein, these quantities are related to each other for $T \ll M_{\text {kin }}$, in particular $D=2 T^{2} / \kappa$ and $\eta=\kappa /\left(2 M_{\text {kin }} T\right)$. Which of the quantities is viewed as "primary" depends on the context: for any mass, $D$ can be expressed through a Kubo relation which in principle permits for a lattice study; in the large-mass limit, $\kappa$ can be expressed through a Kubo relation which permits for a lattice study whose systematic errors should be better under control than for $D$; in the large-mass limit, $\eta$ can be interpreted as a kinetic equilibration rate which leads to a direct physical interpretation (its inverse can be compared with the medium life time).

The challenge with a non-perturbative determination of $D$ is that the corresponding spectral function shows a very narrow transport peak in the large-mass and/or weakcoupling limit, of width $\eta \sim \alpha_{\mathrm{s}}^{2} T^{2} / M[5]$. A controlled reconstruction of a spectral function from imaginary-time data is a hard problem, and practically impossible in the presence of such sharp features. Following a suggestion in ref. [6], the possibility to rather extract $\kappa$ was worked out in ref. [7]. In particular, it was argued that the corresponding spectral function contains no sharp transport peak, being instead flat at small frequencies. This should allow for a somewhat controlled extraction of the transport coefficient, and indeed many measurements have been carried out in recent years [8-13], supplementing LO [14] and NLO [15] perturbative computations.

The definition of $\kappa$ in ref. [7] is related to the standard Kubo relation for the diffusion coefficient, $D$. Whereas $D$ is obtained as a transport coefficient (i.e. height of the transport peak) related to the 2-point correlator of the vector current (which is denoted by $\hat{\mathcal{J}}_{i}$ ), the idea of ref. [7] is to instead consider the tail of the transport peak. Formally, this is obtained by multiplying the vector spectral function by $\omega^{2}$, where $\omega$ is frequency. Inside a Fourier transform, $\omega$ can be converted to a time derivative, so this means that we are really considering the two-point correlator of an "acceleration", $\mathrm{d} \hat{\mathcal{J}}_{i} / \mathrm{d} t$, rather than of a "velocity", $\hat{\mathcal{J}}_{i}$. The correct normalization requires that the whole is multiplied by $M^{2}{ }^{1}$ and divided by the quark number susceptibility, $\chi$, which then led ref. [7] to define

$$
\kappa^{(M)}(\omega) \equiv \frac{1}{3 \chi} \int_{-\infty}^{\infty} \mathrm{d} t e^{i \omega t} \int_{\mathbf{x}}\left\langle\frac{1}{2}\left\{\hat{\mathcal{F}}^{i}(t, \mathbf{x}), \hat{\mathcal{F}}^{i}(0, \mathbf{0})\right\}\right\rangle
$$

where a sum over the spatial indices $i$ is implied, and $\hat{\mathcal{F}}_{i} \equiv M \mathrm{~d} \hat{\mathcal{J}}_{i} / \mathrm{d} t$. It was shown in ref. [7] that the ordered limit $\kappa \equiv \lim _{\omega \rightarrow 0} \lim _{M \rightarrow \infty} \kappa^{(M)}(\omega)$ is an ultraviolet finite observable, which can be defined on the non-perturbative level as a transport coefficient related to the corresponding imaginary-time correlator. In the following, we take eq. (1.1) as a starting point, and inspect the nature of its $\mathcal{O}(T / M)$ corrections.

\footnotetext{
${ }^{1}$ In ref. [7] the multiplication was by the thermally corrected $M_{\text {kin }}^{2}=M^{2}\left\{1+\mathcal{O}\left(\alpha_{\mathrm{s}}^{3 / 2} T / M\right)\right\}$, however the $\mathcal{O}\left(\alpha_{\mathrm{s}}^{3 / 2} T / M\right)$ corrections were not treated, so one could have equally multiplied by $M^{2}$. Here we use $M^{2}$, which does not break Lorentz invariance. The overall normalization of $\kappa$ is fixed later while matching onto a low-energy description, via eqs. (2.3) and (2.9).
} 
This paper is organized as follows. We start with a consideration of Lorentz force correlators within classical electrodynamics (cf. section 2), revealing the key patterns to be confirmed later on in QCD. This is followed by a formal breakdown of eq. (1.1) within the large- $M$ expansion (cf. section 3). The colour-magnetic correlator emerging from these considerations is analyzed perturbatively (cf. section 4), before we conclude with an outlook (cf. section 5).

\section{Classical picture}

Consider the Lorentz force acting on a probe particle of momentum $\mathbf{p}$ and charge $q$ :

$$
\dot{\mathbf{p}}=q(\mathbf{E}+\mathbf{v} \times \mathbf{B})(t) \equiv \mathbf{F}(t) .
$$

We now imagine a statistical environment, in which the velocities come from a thermal distribution. Given the particle's large inertia, the time scale of the variation of velocities is larger than the time scale of the variation of the electric and magnetic field strengths. This slow evolution is expected to be described by the Langevin equation,

$$
\dot{\mathbf{p}}-\eta \mathbf{p}=\mathbf{f}(t), \quad\left\langle f_{i}(t)\right\rangle=0, \quad\left\langle f_{i}\left(t^{\prime}\right) f_{j}(t)\right\rangle=\kappa \delta_{i j} \delta\left(t-t^{\prime}\right) .
$$

The dissipative coefficient $\eta$ and the noise self-correlator $\kappa$ are related by the fluctuationdissipation theorem, following from the fact that the solution of the Langevin equation satisfies

$$
\lim _{t \rightarrow \infty}\left\langle\mathbf{p}^{2}(t)\right\rangle=\frac{3 \kappa}{2 \eta} .
$$

Furthermore there is a dispersive correction, meaning that the mass implicit to eq. (2.2), $M_{\text {kin }}$, differs from the vacuum mass implicit to eq. (2.1), $M$. The goal now is to extract the "low-energy parameters" $\kappa$ and $\eta$ from properties of the microscopic force in eq. (2.1), which involves a number of steps, enumerated as follows.

(i). The first step is to compare the right-hand sides of eqs. (2.1) and (2.2). Given the different time scales of evolution, statistical averages factorize into averages of velocities and averages of fields, ${ }^{2}$ in particular

$$
\left\langle F_{i}\left(t^{\prime}\right) F_{j}(t)\right\rangle=q^{2}\left\{\left\langle E_{i}\left(t^{\prime}\right) E_{j}(t)\right\rangle+\frac{1}{3}\left\langle\mathbf{v}^{2}\right\rangle\left\langle\delta_{i j} B_{k}\left(t^{\prime}\right) B_{k}(t)-B_{j}\left(t^{\prime}\right) B_{i}(t)\right\rangle\right\} .
$$

Matching with eq. (2.2), $\kappa$ can be extracted as a transport coefficient,

$$
\kappa=\lim _{\omega \rightarrow 0} \int_{-\infty}^{\infty} \mathrm{d} t^{\prime} e^{i \omega\left(t^{\prime}-t\right)} \frac{1}{3} \sum_{i}\left\langle F_{i}\left(t^{\prime}\right) F_{i}(t)\right\rangle .
$$

According to eq. (2.4), the leading term originates from the colour-electric correlator [6], and the first correction from a colour-magnetic one, whose contribution is suppressed by $\left\langle\mathbf{v}^{2}\right\rangle \sim \mathcal{O}(T / M)$ according to eq. (2.9).

\footnotetext{
${ }^{2}$ In order to avoid clutter we denote all statistical averages by $\langle\ldots\rangle$, even if the weight with respect to which the average is taken differs from context to context. In point (ii) we return to certain subtleties concerning the circumstances under which the factorization of averages applies.
} 
(ii). Actually, the argument above is a bit sloppy, as the spatial positions of the fields are suppressed. In reality, if a heavy particle starts from $\mathbf{x}=\mathbf{0}$ at time $t=0$ and has the velocity $\mathbf{v}$, at a later time $t$ it is at $\mathbf{x}(t)=\mathbf{v} t+\mathcal{O}\left(\dot{\mathbf{v}} t^{2}\right)$. Then we should really insert fields as $E_{i}(t, \mathbf{x}(t))=E_{i}(t, \mathbf{0})+t v_{j} \partial_{j} E_{i}(t, \mathbf{0})+\ldots$, and similarly for $B_{i}(t, \mathbf{x}(t))$. For instance, when correlated with $\mathbf{v} \times \mathbf{B}$, the next-to-leading terms in the expansion of $E_{i}$ lead to further effects of $\left\langle\mathbf{v}^{2}\right\rangle$, however multiplied by powers of $t$. Such corrections may be called secular terms. Any practical study should be formulated such that secular terms are avoided, ${ }^{3}$ and we have to watch out for them in section 3 as well.

(iii). There is a further subtlety, related to the difference between non-relativistic and relativistic momenta. Suppose that we consider a non-relativistic momentum $M \mathbf{v}$ rather than a relativistic one, $\mathbf{p} \equiv M \mathbf{u}$, with $\mathbf{u} \equiv \gamma \mathbf{v}$ (the reason for this should become apparent around eq. (3.5)). Writing $\mathbf{v}=\gamma^{-1} \mathbf{u}$ we find

$$
\dot{v}_{i}=\gamma^{-1} \dot{u}_{i}-u_{i} u_{j} \dot{v}_{j}
$$

Moving the last term to the left-hand side, and solving the matrix equation for $\dot{v}_{i}$, leads to

$$
\dot{v}_{i}=\left(\delta_{i j}-v_{i} v_{j}\right) \gamma^{-1} \dot{u}_{j}
$$

Taking the 2-point correlator of these accelerations, and expanding to second order in small velocities, gives

$$
M^{2}\left\langle\dot{v}_{i}\left(t^{\prime}\right) \dot{v}_{j}(t)\right\rangle=\left\langle F_{i}\left(t^{\prime}\right) F_{j}(t)\right\rangle-\left\langle\mathbf{v}^{2} \delta_{i k} \delta_{j l}+v_{i} v_{k} \delta_{j l}+\delta_{i k} v_{j} v_{l}\right\rangle\left\langle F_{k}\left(t^{\prime}\right) F_{l}(t)\right\rangle+\mathcal{O}\left(v^{4}\right) .
$$

Here the force-force correlator can be inserted from eq. (2.4). In order to avoid the second term in eq. (2.8), we should use relativistic momenta.

(iv). Finally, we have to consider the relation of $\kappa$ and $\eta$, originating from eq. (2.3). Equipartition in classical statistical physics implies $\left\langle p_{i} \partial H / \partial p_{i}\right\rangle=T$, where $H$ is the Hamiltonian and no sum over $i$ is taken. For a 1-particle Hamiltonian this corresponds to $\langle\mathbf{p} \cdot \mathbf{v}\rangle=3 T$. Inserting a covariant momentum, with the mass including a dispersive correction as we are now within the low-energy effective description, leads to

$$
\left\langle\gamma \mathbf{v}^{2}\right\rangle=\frac{3 T}{M_{\text {kin }}} .
$$

Up to next-to-leading order in velocities, we may expand $\left\langle\gamma \mathbf{v}^{2}\right\rangle \approx\left\langle\mathbf{v}^{2}+\left(\mathbf{v}^{2}\right)^{2} / 2\right\rangle$. For the quartic part, we can take a Gaussian average, $\left\langle\left(\mathbf{v}^{2}\right)^{2}\right\rangle \approx \frac{5}{3}\left\langle\mathbf{v}^{2}\right\rangle^{2}$. Inserting into eq. (2.9), leads to $\left\langle\mathbf{v}^{2}\right\rangle \approx\left(3 T / M_{\text {kin }}\right)\left\{1-5 T /\left(2 M_{\text {kin }}\right)\right\},{ }^{4}$ and this then gives $\left\langle\mathbf{p}^{2}\right\rangle \approx 3 M_{\text {kin }} T\{1+$

\footnotetext{
${ }^{3}$ In a Langevin simulation (cf., e.g., ref. [14]), this is done by tracking separately the positions and momenta of the heavy quarks: $\mathrm{d} \mathbf{x}=\mathbf{v} \mathrm{d} t, \mathrm{~d} \mathbf{p}=(-\eta \mathbf{p}+\mathbf{f}) \mathrm{d} t$, with $\mathbf{v}=\mathbf{p} / \sqrt{\mathbf{p}^{2}+M_{\mathrm{kin}}^{2}}$. In this case, $\mathbf{f}$ can be generated from a random ensemble applicable to the "old" position $\mathbf{x}$.

${ }^{4}$ At leading order in $\alpha_{\mathrm{s}},\left\langle\mathbf{v}^{2}\right\rangle$ can also be defined as the area under the transport peak in the correlator $\left\langle\hat{\mathcal{J}}_{i} \hat{\mathcal{J}}_{i}\right\rangle$, normalized to the susceptibility $\chi$ [7]. From eqs. (3.4), (3.5) of ref. [16], this leads to $\left\langle\mathbf{v}^{2}\right\rangle=$ $\int_{\mathbf{p}}\left(p / E_{p}\right)^{2} n_{\mathrm{F}}^{\prime}\left(E_{p}\right) / \int_{\mathbf{p}} n_{\mathrm{F}}^{\prime}\left(E_{p}\right)$, where $E_{p}=\sqrt{p^{2}+M^{2}}$ and $n_{\mathrm{F}}$ is the Fermi distribution. After an expansion in $T / M$ up to next-to-leading order, this agrees with the result we cite here.
} 
$\left.5 T /\left(2 M_{\text {kin }}\right)\right\}$. In combination with eq. (2.3), we finally find

$$
\eta \approx \frac{\kappa}{2 M_{\mathrm{kin}} T}\left(1-\frac{5 T}{2 M_{\mathrm{kin}}}\right) .
$$

To summarize, the force felt by a non-relativistic probe particle experiences four types of corrections ${ }^{5}$ at first order in $\left\langle\mathbf{v}^{2}\right\rangle \ll 1$. Summing over the indices, the first correction is from $\left\langle B_{i}\left(t^{\prime}\right) B_{i}(t)\right\rangle$, weighted by $\frac{2}{3}\left\langle\mathbf{v}^{2}\right\rangle$ according to eq. (2.4). The second correction are secular terms, of the type discussed under point (ii). The third correction is from $\left\langle E_{k}\left(t^{\prime}\right) E_{l}(t)\right\rangle$, weighted by $-\left\langle\mathbf{v}^{2} \delta_{i k} \delta_{j l}+v_{i} v_{k} \delta_{j l}+\delta_{i k} v_{j} v_{l}\right\rangle=-\frac{5}{3}\left\langle\mathbf{v}^{2}\right\rangle \delta_{i k} \delta_{j l}$ according to eq. (2.8). This a "trivial" effect, inhibiting acceleration towards the speed of light, and eliminated simply by going from $M \mathbf{v}$ back into the covariant momentum $\mathbf{p}$. The fourth correction is to the fluctuation-dissipation relation, according to eq. (2.10). Finally, there is a dispersive effect, substituting the vacuum mass $M$ through a thermally corrected $M_{\text {kin }}$.

\section{Formal derivation of the force-force correlator}

Motivated by the discussion of the previous section and in particular eq. (2.4), the goal now is to derive an expression for the "microscopic" force-force correlator in QCD.

\subsection{Action up to $\mathcal{O}\left(1 / M^{3}\right)$}

Let $\theta$ be a $2 N_{\mathrm{c}}$-component non-relativistic spinor; $g$ a gauge coupling; $D_{\mu} \equiv \partial_{\mu}-i g A_{\mu}$ a covariant derivative; $g E_{i} \equiv i\left[D_{0}, D_{i}\right]$ a colour-electric field; $g B_{i} \equiv \frac{i}{2} \epsilon_{i j k}\left[D_{j}, D_{k}\right]$ a colourmagnetic field; and $\sigma_{i}$ the Pauli matrices. Starting from the Minkowskian QCD action for one heavy quark flavour, $S_{M}=\int_{\mathcal{X}} \bar{\psi}\left(i \gamma^{\mu} D_{\mu}-M\right) \psi$, where $\int_{\mathcal{X}} \equiv \int \mathrm{d} t \int \mathrm{d}^{3} \mathbf{x}$, and carrying out a standard tree-level computation, yields the expansion

$$
\begin{aligned}
S_{M} \supset \int_{\mathcal{X}} & \theta^{\dagger}\left\{i D_{0}-M+\frac{\mathbf{D}^{2}+g \sigma \cdot \mathbf{B}}{2 M}-\frac{g[\mathbf{D} \cdot \mathbf{E}]+i g \sigma \cdot(\mathbf{D} \times \mathbf{E}-\mathbf{E} \times \mathbf{D})}{8 M^{2}}\right. \\
& \left.+\frac{\mathbf{D}^{4}+g\left\{\mathbf{D}^{2}, \sigma \cdot \mathbf{B}\right\}+g^{2}\left(\mathbf{B}^{2}-\mathbf{E}^{2}\right)+i g^{2} \sigma \cdot(\mathbf{B} \times \mathbf{B}-\mathbf{E} \times \mathbf{E})}{8 M^{3}}+\mathcal{O}\left(\frac{1}{M^{4}}\right)\right\} \theta,
\end{aligned}
$$

where the antiparticle part has been omitted. At the quantum level, further operators get generated and the coefficients of the operators get corrected [17]. Such results are of no direct use to us, however, given that the time derivative in section 3.2 is a short-distance operation and thus changes quantum corrections. We note, furthermore, that the rest mass is often omitted from eq. (3.1), but we keep it visible as a residual mass, as the corresponding Boltzmann factor plays an important role at finite temperature. With these specifications, eq. (3.1) serves as the starting point of our investigation.

\footnotetext{
${ }^{5}$ It is unclear to us whether the corrections discussed could be related to a generalization of the Langevin equation used for describing heavy quarks having relativistic (i.e. non-equilibrated) momenta with respect to the medium. It has been suggested that in this case the "comoving" forces could be correlated as [14]

$$
\left\langle f_{i}\left(t^{\prime}\right) f_{j}(t)\right\rangle \simeq\left\{\left(\delta_{i j}-\hat{p}_{i} \hat{p}_{j}\right) \kappa_{\mathrm{T}}(p)+\hat{p}_{i} \hat{p}_{j} \kappa_{\mathrm{L}}(p)\right\} \delta\left(t-t^{\prime}\right),
$$

where $\hat{p}_{i} \equiv p_{i} / p$. In our equilibrated case, considering local forces as described in footnote 3 , the correlator is proportional to $\delta_{i j}$, implying effectively that $\kappa_{\mathrm{L}}(p)=\kappa_{\mathrm{T}}(p)=\kappa$, where $\kappa$ includes corrections of $\mathcal{O}(T / M)$.
} 


\subsection{Lorentz force up to $\mathcal{O}\left(1 / M^{2}\right)$}

The goal now is to derive the Lorentz force originating from eq. (3.1). Viewing $\theta$ and $\theta^{*}$ as independent fields, the Noether current can be defined as

$$
\mathcal{J}^{\mu} \equiv i\left[\theta_{\alpha r}^{*} \frac{\delta}{\delta\left(\partial_{\mu} \theta_{\alpha r}^{*}\right)}-\theta_{\alpha r} \frac{\delta}{\delta\left(\partial_{\mu} \theta_{\alpha r}\right)}\right] S_{M}
$$

where $\alpha \in\left\{1, \ldots, N_{\mathrm{c}}\right\}$ labels colour and $r \in\{1,2\}$ spin. This yields the components

$$
\begin{aligned}
\mathcal{J}^{0} & =\theta^{\dagger} \theta \\
\mathcal{J}^{i} & =\frac{i \theta^{\dagger}\left(\overleftarrow{D}_{i}-\vec{D}_{i}\right) \theta}{2 M}+\frac{g \epsilon_{i j k} \theta^{\dagger} \sigma_{j} E_{k} \theta}{4 M^{2}}-\frac{i \theta^{\dagger}\left\{D_{i}, \mathbf{D}^{2}+g \sigma \cdot \mathbf{B}\right\} \theta}{4 M^{3}}+\mathcal{O}\left(\frac{1}{M^{4}}\right)
\end{aligned}
$$

Before proceeding, let us note that if we consider a spinless 1-particle plane wave, $\theta \simeq Z e^{-i E t+i \mathbf{p} \cdot \mathbf{x}}, \theta^{\dagger} \simeq Z^{\dagger} e^{i E t-i \mathbf{p} \cdot \mathbf{x}}$, then the on-shell point of eq. (3.1) corresponds to the relation $E=M+\frac{p^{2}}{2 M}-\frac{p^{4}}{8 M^{3}}+\ldots=\sqrt{p^{2}+M^{2}}$. The Noether charge density in eq. (3.3) evaluates to $\mathcal{J}^{0} \simeq|Z|^{2}$, and the current in eq. (3.4) to

$$
\mathcal{J}^{i} \simeq|Z|^{2}\left(\frac{p_{i}}{M}-\frac{p_{i} p^{2}}{2 M^{3}}+\ldots\right)=\frac{|Z|^{2} p_{i}}{\sqrt{p^{2}+M^{2}}}=|Z|^{2} v_{i} .
$$

Therefore, $\mathcal{J}^{i} / \mathcal{J}^{0}$ represents the velocity rather than the covariant velocity, and we expect the argument around eqs. (2.7) and (2.8) to apply to considerations following from eq. (1.1).

To obtain the Lorentz force, we take a time derivative of the current, and use equations of motion. As the observable in eq. (1.1) involves a spatial average, partial integrations are permitted in spatial directions. Obviously the Noether charge $\int_{\mathbf{x}} \mathcal{J}^{0}$ is conserved, but the spatial currents are not, obeying

$$
\begin{aligned}
\int_{\mathbf{x}} M \partial_{0} \mathcal{J}^{i}=\int_{\mathbf{x}} \theta^{\dagger}\{ & -g E_{i}+\frac{\left[D_{i}, \mathbf{D}^{2}+g \sigma \cdot \mathbf{B}\right]}{2 M}+\frac{g\left[D_{0}, \sigma \times \mathbf{E}\right]_{i}}{4 M} \\
& -\frac{g\left[D_{i},[\mathbf{D} \cdot \mathbf{E}]+i \sigma \cdot(\mathbf{D} \times \mathbf{E}-\mathbf{E} \times \mathbf{D})\right]+i g\left[\mathbf{D}^{2}+g \sigma \cdot \mathbf{B}, \sigma \times \mathbf{E}\right]_{i}}{8 M^{2}} \\
& \left.-\frac{i\left[D_{0},\left\{D_{i}, \mathbf{D}^{2}+g \sigma \cdot \mathbf{B}\right\}\right]}{4 M^{2}}+\mathcal{O}\left(\frac{1}{M^{3}}\right)\right\} \theta
\end{aligned}
$$

Let us stress again that at the quantum level, further operators and non-trivial Wilson coefficients are generated, but here we remain at the tree level.

In view of a lattice study or thermal field theory computation, the final step is to Wick rotate the result to Euclidean signature: $D_{0} \rightarrow i D_{0}, E_{i} \rightarrow i E_{i}$. Furthermore we reduce the number of explicit derivatives appearing in eq. (3.6), by making use of

$$
\begin{aligned}
{\left[D_{i}, \mathbf{D}^{2}\right] } & =-i g(\mathbf{D} \times \mathbf{B}-\mathbf{B} \times \mathbf{D})_{i} \\
{\left[D_{0},\left\{D_{i}, \mathbf{D}^{2}\right\}\right] } & =-i g\left(E_{i} \vec{D}^{2}+\overleftarrow{D}^{2} E_{i}+E_{j} \vec{D}_{j} \vec{D}_{i}+\overleftarrow{D}_{i} \overleftarrow{D}_{j} E_{j}-\overleftarrow{D}_{j} E_{j} \vec{D}_{i}-\overleftarrow{D}_{i} E_{j} \vec{D}_{j}\right)
\end{aligned}
$$

Lowering the index $i$ on the left-hand side of eq. (3.6), in order to insert an overall minus sign, but suppressing it from the notation, the Lorentz force operators originating from 
eq. (3.6) can then be enumerated as

$$
\begin{aligned}
O_{0} & \equiv \theta^{\dagger} i g E_{i} \theta \\
O_{1 a} & \equiv \frac{\theta^{\dagger} i g(\mathbf{D} \times \mathbf{B}-\mathbf{B} \times \mathbf{D})_{i} \theta}{2 M}, \\
O_{1 b} & \equiv \frac{\theta^{\dagger}(-g)\left[D_{i}, \sigma \cdot \mathbf{B}\right] \theta}{2 M} \\
O_{1 c} & \equiv \frac{\theta^{\dagger} g\left[D_{0}, \sigma \times \mathbf{E}\right]_{i} \theta}{4 M}, \\
O_{2 a} & \equiv \frac{\theta^{\dagger} i g\left[D_{i},[\mathbf{D} \cdot \mathbf{E}]\right] \theta}{8 M^{2}} \\
O_{2 b} & \equiv \frac{\theta^{\dagger}(-g)\left[D_{i}, \sigma \cdot(\mathbf{D} \times \mathbf{E}-\mathbf{E} \times \mathbf{D})\right] \theta}{8 M^{2}} \\
O_{2 c} & \equiv \frac{\theta^{\dagger} g\left[\sigma \times \mathbf{E}, \mathbf{D}^{2}\right]_{i} \theta}{8 M^{2}}, \\
O_{2 d} & \left.\equiv \frac{\theta^{\dagger} g^{2}[\sigma \times \mathbf{E}, \sigma \cdot \mathbf{B}]_{i} \theta}{8 M^{2}}, \overleftarrow{D^{2}}, \overleftarrow{D}^{2} E_{i}+E_{j} \vec{D}_{j} \vec{D}_{i}+\overleftarrow{D}_{i} \overleftarrow{D}_{j} E_{j}-\overleftarrow{D}_{j} E_{j} \vec{D}_{i}-\overleftarrow{D}_{i} E_{j} \vec{D}_{j}\right) \theta \\
O_{2 e} & \equiv \frac{\theta^{\dagger} i g\left(E_{i} \vec{D}^{2}\right.}{4 M^{2}} \\
O_{2 f} & \equiv \frac{\theta^{\dagger}(-g)\left[D_{0},\left\{D_{i}, \sigma \cdot \mathbf{B}\right\}\right] \theta}{.}
\end{aligned}
$$

The Euclidean action with respect to which thermal averages are taken $\left(e^{i S_{M}} \rightarrow e^{-S_{E}}\right)$ becomes

$$
\begin{aligned}
S_{E} & =S_{0}+S_{1}+S_{2}+\mathcal{O}\left(\frac{1}{M^{3}}\right), \\
S_{0} & =\int_{X} \theta^{\dagger}\left(D_{0}+M\right) \theta, \\
S_{1} & =-\int_{X} \frac{\theta^{\dagger}\left(\mathbf{D}^{2}+g \sigma \cdot \mathbf{B}\right) \theta}{2 M}, \\
S_{2} & =\int_{X} \frac{\theta^{\dagger}(i g)[\mathbf{D} \cdot \mathbf{E}] \theta-\theta^{\dagger} g \sigma \cdot(\mathbf{D} \times \mathbf{E}-\mathbf{E} \times \mathbf{D}) \theta}{8 M^{2}},
\end{aligned}
$$

where $\int_{X} \equiv \int_{0}^{\beta} \mathrm{d} \tau \int_{\mathbf{x}}$. We note that in normal HQET, only $S_{0}$ appears as a weight in the thermal average, whereas $S_{1}$ and $S_{2}$ are expanded into correlation functions, however in the thermal context taking this limit requires care, as will be discussed in section 3.4.

\subsection{Force-force correlator up to $\mathcal{O}\left(1 / M^{2}\right)$}

Let us consider the 2-point correlation function of the operator defined as a sum of eqs. (3.9)-(3.18). As alluded to at the end of section 3.2, we do not expand the action in powers of $1 / M$ yet, i.e. we are working within the NRQCD rather than HQET action for a moment.

After inserting the operators, we carry out Wick contractions for the fermion fields. A fermion propagator (which is a $2 N_{\mathrm{c}} \times 2 N_{\mathrm{c}}$-matrix) is defined as

$$
\Delta\left(\tau_{2}, \mathbf{y} ; \tau_{1}, \mathbf{x}\right) \equiv\left\langle\theta\left(\tau_{2}, \mathbf{y}\right) \theta^{\dagger}\left(\tau_{1}, \mathbf{x}\right)\right\rangle
$$


and covariant derivatives acting on it as

$$
\vec{D}_{i} \vec{D}_{j} \ldots \Delta\left(\tau_{2}, \mathbf{y} ; \tau_{1}, \mathbf{x}\right) \ldots \overleftarrow{D}_{k} \overleftarrow{D}_{l} \equiv \Delta_{i j \ldots ; \ldots k l}\left(\tau_{2}, \mathbf{y} ; \tau_{1}, \mathbf{x}\right)
$$

Correlators are denoted by

$$
G_{m n}(\tau) \equiv \frac{\sum_{i=1}^{3} \int_{\mathbf{x}}\left\langle O_{m}(\tau, \mathbf{x}) O_{n}(0, \mathbf{0})\right\rangle}{3 \chi}, \quad G_{\{m n\}}(\tau) \equiv G_{m n}(\tau)+G_{n m}(\tau)
$$

where the quark number susceptibility reads

$$
\chi \equiv \int_{\mathbf{x}}\left\langle\theta^{\dagger} \theta(\tau, \mathbf{x}) \theta^{\dagger} \theta(0, \mathbf{0})\right\rangle, \quad \tau>0 .
$$

The value of $\chi$ is independent of the choice of $\tau$, because $\int_{\mathbf{x}} \theta^{\dagger} \theta$ is a conserved charge within the NRQCD/HQET action.

At leading order in the $1 / M$-expansion, i.e. with the operator from eq. (3.9), the definitions above lead to

$$
G_{00}(\tau)=-\frac{g^{2}}{3 \chi} \int_{\mathbf{x}} \operatorname{Tr}\left\langle\Delta(\beta, \mathbf{0} ; \tau, \mathbf{x}) E_{i}(\tau, \mathbf{x}) \Delta(\tau, \mathbf{x} ; 0, \mathbf{0}) E_{i}(0, \mathbf{0})\right\rangle
$$

where a sum over the index $i$ is implied.

Proceeding to $\mathcal{O}(1 / M)$, we are faced with the correlators $G_{\{01 a\}}, G_{\{01 b\}}$, and $G_{\{01 c\}}$. Given that the operators $O_{1 b}$ and $O_{1 c}$ contain a Pauli matrix and that spin effects only appear at $\mathcal{O}(1 / M)$ in eq. (3.21), the latter two vanish up to $\mathcal{O}\left(1 / M^{2}\right)$. The remaining one gives

$$
\begin{aligned}
G_{\{01 a\}}(\tau)=\frac{g^{2} \epsilon_{i j k}}{6 M \chi} \int_{\mathbf{x}} \operatorname{Tr}\langle & \Delta(\beta, \mathbf{0} ; \tau, \mathbf{x}) E_{i}(\tau, \mathbf{x}) \Delta_{; j}(\tau, \mathbf{x} ; 0, \mathbf{0}) B_{k}(0, \mathbf{0}) \\
& -\Delta_{j ;}(\beta, \mathbf{0} ; \tau, \mathbf{x}) E_{i}(\tau, \mathbf{x}) \Delta(\tau, \mathbf{x} ; 0, \mathbf{0}) B_{k}(0, \mathbf{0}) \\
& +\Delta_{; j}(\beta, \mathbf{0} ; \tau, \mathbf{x}) B_{k}(\tau, \mathbf{x}) \Delta(\tau, \mathbf{x} ; 0, \mathbf{0}) E_{i}(0, \mathbf{0}) \\
& \left.-\Delta(\beta, \mathbf{0} ; \tau, \mathbf{x}) B_{k}(\tau, \mathbf{x}) \Delta_{j ;}(\tau, \mathbf{x} ; 0, \mathbf{0}) E_{i}(0, \mathbf{0})\right\rangle .
\end{aligned}
$$

We return to a discussion of this correlator in section 3.4.

Finally, at order $1 / M^{2}$, the correlators are $G_{1 a 1 a}, G_{1 b 1 b}, G_{1 c 1 c}, G_{\{1 a 1 b\}}, G_{\{1 a 1 c\}}, G_{\{1 b 1 c\}}$, $G_{\{02 a\}}, G_{\{02 b\}}, G_{\{02 c\}}, G_{\{02 d\}}, G_{\{02 e\}}$, and $G_{\{02 f\}}$. Among these, $G_{\{1 a 1 b\}}, G_{\{1 a 1 c\}}, G_{\{02 b\}}$, $G_{\{02 c\}}$, and $G_{\{02 f\}}$ are really of $\mathcal{O}\left(1 / M^{3}\right)$, because only one of the operators contains a Pauli matrix, and spin-dependent effects are suppressed by $1 / M$ in the action, cf. eq. (3.21). Among the rest, $G_{1 b 1 b}, G_{1 c 1 c}, G_{\{1 b 1 c\}}, G_{\{02 a\}}$, and $G_{\{02 d\}}$ are of $\mathcal{O}\left(1 / M^{2}\right)$. The two remaining ones, $G_{1 a 1 a}$ and $G_{\{02 e\}}$, are the most important ones, as they contain derivatives acting on the heavy quark propagators that cannot be eliminated by partial integrations. As discussed in section 3.4, these two correlators are really of $\mathcal{O}(T / M)$, and deserve to be 
given explicitly:

$$
\begin{aligned}
G_{1 a 1 a}(\tau)=-\frac{g^{2} \epsilon_{i j k} \epsilon_{i m n}}{12 M^{2} \chi} \int_{\mathbf{x}} \operatorname{Tr}\left\langle\Delta_{; j}(\beta, \mathbf{0} ; \tau, \mathbf{x}) B_{k}(\tau, \mathbf{x}) \Delta_{; m}(\tau, \mathbf{x} ; 0, \mathbf{0}) B_{n}(0, \mathbf{0})\right. \\
+\Delta_{n ; j}(\beta, \mathbf{0} ; \tau, \mathbf{x}) B_{k}(\tau, \mathbf{x}) \Delta(\tau, \mathbf{x} ; 0, \mathbf{0}) B_{m}(0, \mathbf{0}) \\
+\Delta(\beta, \mathbf{0} ; \tau, \mathbf{x}) B_{j}(\tau, \mathbf{x}) \Delta_{k ; m}(\tau, \mathbf{x} ; 0, \mathbf{0}) B_{n}(0, \mathbf{0}) \\
\left.\left.+\Delta_{n ;}(\beta, \mathbf{0} ; \tau, \mathbf{x}) B_{j}(\tau, \mathbf{x}) \Delta_{k ;} ; \tau, \mathbf{x} ; 0, \mathbf{0}\right) B_{m}(0, \mathbf{0})\right\rangle, \\
G_{02 a}(\tau)=-\frac{g^{2}}{12 M^{2} \chi} \int_{\mathbf{x}} \operatorname{Tr}\left\langle\Delta_{j j ;}(\beta, \mathbf{0} ; \tau, \mathbf{x}) E_{i}(\tau, \mathbf{x}) \Delta(\tau, \mathbf{x} ; 0, \mathbf{0}) E_{i}(0, \mathbf{0})\right. \\
+\Delta(\beta, \mathbf{0} ; \tau, \mathbf{x}) E_{i}(\tau, \mathbf{x}) \Delta_{; j j}(\tau, \mathbf{x} ; 0, \mathbf{0}) E_{i}(0, \mathbf{0}) \\
+\Delta_{j i ;}(\beta, \mathbf{0} ; \tau, \mathbf{x}) E_{i}(\tau, \mathbf{x}) \Delta(\tau, \mathbf{x} ; 0, \mathbf{0}) E_{j}(0, \mathbf{0}) \\
+\Delta(\beta, \mathbf{0} ; \tau, \mathbf{x}) E_{i}(\tau, \mathbf{x}) \Delta_{; i j}(\tau, \mathbf{x} ; 0, \mathbf{0}) E_{j}(0, \mathbf{0}) \\
-\Delta_{i ;}(\beta, \mathbf{0} ; \tau, \mathbf{x}) E_{i}(\tau, \mathbf{x}) \Delta_{; j}(\tau, \mathbf{x} ; 0, \mathbf{0}) E_{j}(0, \mathbf{0}) \\
\left.-\Delta_{j ;}(\beta, \mathbf{0} ; \tau, \mathbf{x}) E_{i}(\tau, \mathbf{x}) \Delta_{; i}(\tau, \mathbf{x} ; 0, \mathbf{0}) E_{j}(0, \mathbf{0})\right\rangle
\end{aligned}
$$

In eq. (3.30) we have displayed only one ordering, with the other one in $G_{\{02 a\}}=G_{02 a}+G_{2 a 0}$ giving a similar result.

\subsection{Physical effects up to $\mathcal{O}(T / M)$}

In a vacuum setting, when heavy quarks are bound inside mesons, their momenta are balanced against those of the light constituents, and thus of order $\Lambda_{\overline{\mathrm{MS}}}$ or $m_{\pi}$. In this case, the counting of powers of $1 / M$ is simple: the explicit terms appearing in the denominator represent also the true suppression factors. The situation changes at high temperatures $T \gg \Lambda_{\overline{\mathrm{MS}}}$, because thermal kicks can give the heavy quarks large momenta. In fact, in the asymptotic limit of small $\alpha_{\mathrm{s}}$, equipartition asserts that heavy-quark momenta are of order $p^{2} \sim M T$. Therefore we should count covariant derivatives acting on heavy quark fields as $p \sim \sqrt{M T}$, and heavy quark velocities as shown in eq. (2.9).

Given that $p \sim \sqrt{M T} \gg T \gtrsim \Lambda_{\overline{\mathrm{MS}}}$, the spatial momenta of the heavy quarks should be "integrated out", if we want to arrive at a HQET type formulation for studying nonperturbative effects from the light parton sector. As a result of the integration, we should be left over with HQET type correlation functions, multiplied by Wilson coefficients that account for the effects of the hard modes. Specifically, we may expect $\left\langle\mathbf{v}^{2}\right\rangle$ to play the role of a (tree-level) Wilson coefficient, which would be modified by a multiplicative correction at the loop level. ${ }^{6}$

\footnotetext{
${ }^{6}$ The Wilson coefficient generically displays a non-vanishing anomalous dimension, corresponding to that of the correlation function that it multiplies. Its determination entails two matching steps: first, between full QCD and the non-relativistic operator in eq. (3.10); second, between thermal NRQCD and thermal HQET from which the momenta $p \sim \sqrt{M T}$ have been integrated out.
} 
For a concrete leading-order implementation of this ideology, we note that in the static limit, i.e. with the action from eq. (3.20), the heavy quark propagators reduce to $(\tau>0)$

$$
\begin{aligned}
& \left\langle\theta_{\alpha s}(\tau, \mathbf{x}) \theta_{\beta r}^{*}(0, \mathbf{y})\right\rangle \stackrel{M \rightarrow \infty}{=} U_{\alpha \beta}(\tau ; 0) \delta_{s r} e^{-\tau M} \lim _{M \rightarrow \infty} \int_{\mathbf{p}} e^{i \mathbf{p} \cdot(\mathbf{x}-\mathbf{y})-\tau \epsilon_{p}}, \\
& \left\langle\theta_{\alpha s}(\beta, \mathbf{y}) \theta_{\beta r}^{*}(\tau, \mathbf{x})\right\rangle \stackrel{M \rightarrow \infty}{=} U_{\alpha \beta}(\beta ; \tau) \delta_{s r} e^{(\tau-\beta) M} \lim _{M \rightarrow \infty} \int_{\mathbf{q}} e^{i \mathbf{q} \cdot(\mathbf{y}-\mathbf{x})+(\tau-\beta) \epsilon_{q}},
\end{aligned}
$$

where $U$ is a Wilson line in the fundamental representation and $\epsilon_{p} \equiv p^{2} /(2 M)$ from eq. (3.21). Derivatives acting on the propagators yield powers of $\mathbf{p}$ or $\mathbf{q}$, up to radiative corrections from short-distance gauge field fluctuations. If we denote by $\mathbf{k}$ the momentum transfer from the magnetic or electric field insertion (so that $\mathbf{q}=\mathbf{p}+\mathbf{k}$ ) and shift $\mathbf{p}$ by $-\mathbf{k} / 2$ for maximal symmetry, then the two propagators from eqs. (3.31) and (3.32) combine into an exponential

$$
(\tau-\beta) \epsilon_{\mathbf{p}+\mathbf{k} / 2}-\tau \epsilon_{\mathbf{p}-\mathbf{k} / 2}=-\beta\left(\epsilon_{p}+\frac{k^{2}}{8 M}\right)+\left(\tau-\frac{\beta}{2}\right) \mathbf{v} \cdot \mathbf{k} .
$$

The part $-\beta \epsilon_{p}$ is of $\mathcal{O}(1)$ and cannot be expanded in, whereas $\beta k^{2} / M \sim T / M \ll 1$ represents a small correction, similar to the Pauli-term in eq. (3.21). The last part of eq. (3.33) generates secular terms in the sense discussed under point (ii) of section 2, with $\tau-\beta / 2$ representing an imaginary-time interval and $\mathbf{k} \leftrightarrow-i \nabla$ generating spatial translations.

Summarizing these considerations, the term in the exponential that cannot be expanded is $-\beta \epsilon_{p}$. We normalize the integral over powers of $p_{i}$ by the same factor appearing in the denominator (i.e. $\chi$ ),

$$
\left\langle p_{i} p_{j} \ldots\right\rangle \equiv \frac{\lim _{\mathbf{x} \rightarrow \mathbf{0}} \int_{\mathbf{p}} p_{i} p_{j} \ldots e^{i \mathbf{p} \cdot \mathbf{x}-\beta \epsilon_{p}}}{\lim _{\mathbf{x} \rightarrow \mathbf{0}} \int_{\mathbf{p}} e^{i \mathbf{p} \cdot \mathbf{x}-\beta \epsilon_{p}}} .
$$

The Pauli term in eq. (3.21) can be expanded in. Furthermore, in a perturbative integration out of heavy quark momenta, $-\beta k^{2} /(8 T M)$ in eq. (3.33) can be expanded in, whereas the treatment of the last term in eq. (3.33) requires a decision on how to handle secular terms.

Let us now apply this recipe to the denominator,

$$
\chi=\int_{\mathbf{x}}\left\langle\left(\theta^{\dagger} \theta\right)(\tau, \mathbf{x})\left(\theta^{\dagger} \theta\right)(0, \mathbf{0}) e^{-S_{1}-S_{2}-\ldots}\right\rangle_{0}
$$

where $S_{1,2}$ are from eqs. (3.21) and (3.22), respectively, and $\langle\ldots\rangle_{0}$ denotes averaging of the heavy quark fields with respect to $S_{0}$ from eq. (3.20). At leading order we find

$$
\chi_{(0)}=2 e^{-\beta M} \int_{\mathbf{p}} e^{-\beta \epsilon_{p}} \operatorname{Tr}\langle U(\beta ; 0)\rangle .
$$

There is no first-order correction, because the Pauli term from eq. (3.22) vanishes by the spin trace. There are corrections at $1 / M^{2}$, but these are not needed, as we want to extract effects of $\mathcal{O}(T / M)$. In short, $\chi$ can be replaced by $\chi_{(0)}$ at $\mathcal{O}(T / M)$. The same arguments 
apply also in the numerator: at $\mathcal{O}(T / M)$, we do not need to worry about the expansion of the Pauli term in eq. (3.21) nor of eq. (3.22).

With this recipe, the correlator $G_{\{01 a\}}$ from eq. (3.28) of naive $\mathcal{O}(1 / M)$ vanishes at leading order, because it contains one spatial derivative and is thus proportional to $\langle\mathbf{p}\rangle=\mathbf{0}$ or $\langle\mathbf{q}\rangle=\mathbf{0}$. However, if we expand the last term of eq. (3.33) into the correlator, we get a contribution proportional to $\left\langle\mathbf{v}^{2}\right\rangle \sim T / M$ which does not vanish. This contribution contains the prefactor $\tau-\beta / 2$ and is a secular term in the sense discussed under point (ii) of section 2. As explained there, we do not think that it is physically sensible to consider these effects, if the goal is to match onto a Langevin description.

The correlator $G_{\{02 e\}}$ from eq. (3.30) does not vanish, but evaluates at $\mathcal{O}(T / M)$ to

$$
G_{\{02 e\}}(\tau)=\frac{5\left\langle\mathbf{v}^{2}\right\rangle}{3} \frac{g^{2} \operatorname{Tr}\left\langle U(\beta ; \tau) E_{i}(\tau) U(\tau ; 0) E_{i}(0)\right\rangle}{3 \operatorname{Tr}\langle U(\beta ; 0)\rangle} .
$$

We have suppressed spatial coordinates, as they are all the same. This is just $-5\left\langle\mathbf{v}^{2}\right\rangle / 3$ times the leading-order correlator originating from eq. (3.27), and corresponds to the effects discussed below eq. (2.8). As elaborated upon there, the corresponding effects can be eliminated by going over to covariant momenta in the Langevin description.

Finally, there is genuine effect of $\mathcal{O}(T / M)$ from $G_{1 a 1 a}$ in eq. (3.29),

$$
G_{1 a 1 a}(\tau)=\frac{2\left\langle\mathbf{v}^{2}\right\rangle}{3} \frac{g^{2} \operatorname{Tr}\left\langle U(\beta ; \tau) B_{i}(\tau) U(\tau ; 0) B_{i}(0)\right\rangle}{3 \operatorname{Tr}\langle U(\beta ; 0)\rangle} .
$$

Both expectation values are real in the physical ground state, so we may add a real part in order to eliminate noise, and define somewhat more explicitly

$$
G_{B}(\tau) \equiv \frac{\sum_{i} \operatorname{Re} \operatorname{Tr}\left\langle U(\beta ; \tau) g B_{i}(\tau) U(\tau ; 0) g B_{i}(0)\right\rangle}{3 \operatorname{Re} \operatorname{Tr}\langle U(\beta ; 0)\rangle} .
$$

Then the full transport coefficient reads

$$
\kappa_{\mathrm{tot}} \simeq \kappa_{E}+\frac{2}{3}\left\langle\mathbf{v}^{2}\right\rangle \kappa_{B}
$$

where $\left\langle\mathbf{v}^{2}\right\rangle$ is given by eq. (2.9) and the discussion below it, and $\kappa_{E, B}$ are the transport coefficients corresponding to $G_{E, B}$, respectively.

\section{Perturbative evaluation}

The purpose of this section is to look in more detail into the correlator $G_{B}$ defined in eq. (3.39), determining both the temporal correlator and the transport coefficient at leading order.

\subsection{Temporal correlator}

Let us start by recalling the form of the electric correlator from eq. (3.27),

$$
\begin{aligned}
\operatorname{Tr}\left\langle U(\beta ; \tau) E_{i}(\tau) U(\tau ; 0) E_{i}(0)\right\rangle \quad \stackrel{\mathcal{O}}{\stackrel{\left(g^{0}\right)}{=}} \frac{N_{\mathrm{c}}^{2}-1}{2} \oiint_{K} \frac{\left(3 \omega_{n}^{2}+\mathbf{k}^{2}\right) e^{i \omega_{n} \tau}}{\omega_{n}^{2}+\mathbf{k}^{2}} \\
\omega_{n}^{2}=\omega_{n}^{2}+\mathbf{k}^{2}-\mathbf{k}^{2} \\
=\left(N_{\mathrm{c}}^{2}-1\right) \oiint_{K} \frac{\mathbf{k}^{2} e^{i \omega_{n} \tau}}{\omega_{n}^{2}+\mathbf{k}^{2}},
\end{aligned}
$$


where in the second step a contact term vanishing in dimensional regularization was omitted. For $G_{B}$ the corresponding evaluation gives

$$
\begin{aligned}
\operatorname{Tr}\left\langle U(\beta ; \tau) B_{i}(\tau) U(\tau ; 0) B_{i}(0)\right\rangle \stackrel{\left.\mathcal{O} \underline{g}^{0}\right)}{=} \frac{N_{\mathrm{c}}^{2}-1}{2} \oiint_{K} \frac{\epsilon_{i j l} \epsilon_{i m n} k_{j} k_{m} \delta_{l n} e^{i \omega_{n} \tau}}{\omega_{n}^{2}+\mathbf{k}^{2}} \\
=\left(N_{\mathrm{c}}^{2}-1\right) \oiint_{K} \frac{\mathbf{k}^{2} e^{i \omega_{n} \tau}}{\omega_{n}^{2}+\mathbf{k}^{2}} .
\end{aligned}
$$

Recalling the overall minus sign in the colour-electric correlator, cf. eq. (3.27), the correlators $G_{E}$ and $G_{B}$ agree at leading order. Adding the denominator, carrying out the Matsubara sum, and denoting by $G_{B}^{(n)}$ the contribution of order $g^{n}$, we get [7]

$$
\begin{aligned}
G_{B}^{(2)}(\tau) & =\frac{g^{2} C_{\mathrm{F}}}{3} \int_{\mathbf{k}}\left[e^{\tau k}+e^{(\beta-\tau) k}\right] k n_{\mathrm{B}}(k) \\
& =g^{2} C_{\mathrm{F}} \pi^{2} T^{4}\left[\frac{\cos ^{2}(\pi \tau T)}{\sin ^{4}(\pi \tau T)}+\frac{1}{3 \sin ^{2}(\pi \tau T)}\right],
\end{aligned}
$$

where $n_{\mathrm{B}}$ is the Bose distribution. This can serve as a normalization for lattice results.

\subsection{Transport coefficient}

The determination of the transport coefficient $\kappa_{B}$ is non-trivial so we give some details, following the presentation for $G_{E}$ and $\kappa_{E}$ in ref. [18].

\subsubsection{Setup}

Formally, after the Fourier transformation

$$
\tilde{G}_{B}\left(\omega_{n}\right)=\int_{0}^{\beta} \mathrm{d} \tau e^{i \omega_{n} \tau} G_{B}(\tau)
$$

we may extract the spectral function

$$
\rho_{B}(\omega)=\operatorname{Im} \tilde{G}_{B}\left(\omega_{n} \rightarrow-i\left[\omega+i 0^{+}\right]\right) .
$$

The transport coefficient is given by

$$
\kappa_{B}=\lim _{\omega \rightarrow 0} \frac{2 T \rho_{B}(\omega)}{\omega},
$$

where the factor $2 T / \omega$ transforms a spectral function (commutator) into the anticommutator appearing in eq. (1.1).

As is normally the case with transport coefficients, practical computations necessitate a resummation of the perturbative series. We may write

$$
\kappa_{B}=\kappa_{B}^{\mathrm{QCD}, \text { expanded }}-\kappa_{B}^{\mathrm{HTL}, \text { expanded }}+\kappa_{B}^{\mathrm{HTL}, \text { full }} .
$$

Here "expanded" denotes an unresummed computation, i.e. a naive expansion in the coupling $g^{2}$; HTL stands for Hard Thermal Loop resummation [19-22]; and the subtraction 


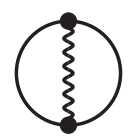

(a)

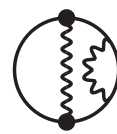

(b)

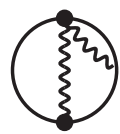

(g)

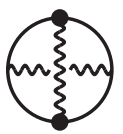

(c)

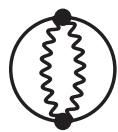

(h)

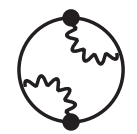

(d)

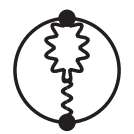

(i)

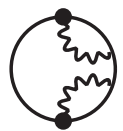

(e)

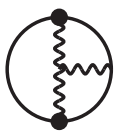

(j)

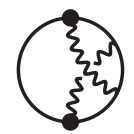

(f)

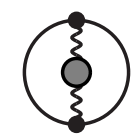

(k)

Figure 1. The LO and NLO graphs contributing to the colour-magnetic correlator, $G_{B}$, defined in eq. (3.39). The big circle denotes a Polyakov loop; the small dots colour-magnetic field strengths; and the grey blob the 1-loop gauge field self-energy.

removes the danger of double counting. If there were no IR divergences, or if we could compute to all orders in the expansion, the latter two terms would cancel against each other.

The full spectral function, computed as in ref. [18], contains a vacuum part proportional to $\omega^{3}$ as well as a thermal part with a complicated functional dependence on $\omega$. According to eq. (4.6), we only need the part linear in $\omega$ at small $\omega$, which can only originate from thermal corrections. Therefore, in the following, we omit those vacuum corrections which are of NLO in the weak-coupling expansion (for completeness we do display the LO vacuum term in eq. (4.10)).

We carry out the computation with dimensional regularization, in $D \equiv 4-2 \epsilon$ dimensions. Introducing a scale parameter $\mu$, the $\overline{\mathrm{MS}}$ scale is defined as $\bar{\mu}^{2} \equiv 4 \pi \mu^{2} e^{-\gamma_{\mathrm{E}}}$. The Levi-Civita-symbol is written as $\epsilon_{i j k} \epsilon_{l m n} \equiv \delta_{i l} \delta_{j m} \delta_{k n}+\delta_{i m} \delta_{j n} \delta_{k l}+\delta_{i n} \delta_{j l} \delta_{k m}-\delta_{i l} \delta_{j n} \delta_{k m}-$ $\delta_{i m} \delta_{j l} \delta_{k n}-\delta_{i n} \delta_{j m} \delta_{k l}$. Often we are faced with $\delta_{i l} \delta_{j m} \epsilon_{i j k} \epsilon_{l m n}=(D-3)(D-2) \delta_{k n}$. Following ref. [18], we denote a massless propagator as

$$
G\left(x_{0}, \mathbf{x}\right) \equiv \oiint_{K} \frac{e^{i\left(k_{n} x_{0}+\mathbf{k} \cdot \mathbf{x}\right)}}{K^{2}} .
$$

\subsubsection{QCD contributions}

We start by extracting the part denoted by $\kappa_{B}^{\mathrm{QCD} \text {,expanded }}$ in eq. (4.7). This originates from graphs that are of NLO, i.e. $\mathcal{O}\left(g^{4}\right)$, with diagrams as shown in figure 1 . We have carried out the computation in a general covariant gauge, verifying its gauge independence; below, graph-by-graph results are listed for the Feynman gauge.

The LO graph gives

$$
\delta_{(\mathrm{a})} G_{B}(\tau)=\frac{g^{2} C_{\mathrm{F}}(D-3)(D-2)}{3}\left(-\nabla^{2}\right) G(\tau, \mathbf{0}) .
$$

This leads to

$$
\delta_{(\mathrm{a})} \rho_{B}(\omega) \stackrel{D=4}{=} \frac{g^{2} C_{\mathrm{F}} \omega^{3}}{6 \pi},
$$

so there is no contribution to $\kappa_{B}$. We also need the NLO correction to the denominator,

$$
\operatorname{Re} \operatorname{Tr}\langle U(\beta ; 0)\rangle=N_{\mathrm{c}}\left\{1-g^{2} C_{\mathrm{F}} \int_{0}^{\beta} \mathrm{d} \tau^{\prime} \int_{0}^{\tau^{\prime}} \mathrm{d} \tau^{\prime \prime} G\left(\tau^{\prime}-\tau^{\prime \prime}, \mathbf{0}\right)\right\} .
$$


Consider next the diagrams (b-f). In Feynman gauge, the diagrams (d,e,f) are absent. The diagrams (b,c) yield a term proportional to $C_{\mathrm{F}}^{2}$, which cancels when the LO term in eq. (4.9) is combined with the NLO correction from eq. (4.11). The combined effect is thus proportional to $N_{\mathrm{c}} C_{\mathrm{F}}$,

$$
\delta_{(\mathrm{a})} G_{B}(\tau) \times \frac{\chi_{\mathrm{LO}}}{\chi_{\mathrm{NLO}}}+\delta_{(\mathrm{b}-\mathrm{f})} G_{B}(\tau)=\frac{g^{4} N_{\mathrm{c}} C_{\mathrm{F}}(D-3)(D-2)}{3} \mathcal{I}_{4}(\tau)+\mathcal{O}\left(g^{6}\right),
$$

where

$$
\mathcal{I}_{4}(\tau) \equiv \oiint_{K} \frac{k^{2} e^{i k_{n} \tau}}{K^{2}} \oiint_{Q^{\prime}} \frac{e^{i q_{n} \tau}-1}{q_{n}^{2} Q^{2}}
$$

was defined in eq. (3.28) of ref. [18].

Proceeding further, graph (g) is absent in Feynman gauge. Graphs (h) and (i) give

$$
\begin{aligned}
\delta_{(\mathrm{h})} G_{B}(\tau) & =\frac{g^{4} N_{\mathrm{c}} C_{\mathrm{F}}(D-3)(D-2)(D-1)}{6} \mathcal{I}_{1}(\tau), \\
\delta_{(\mathrm{i})} G_{B}(\tau) & =-g^{4} N_{\mathrm{c}} C_{\mathrm{F}}(D-3)(D-2) \mathcal{I}_{2}(\tau)
\end{aligned}
$$

where $\mathcal{I}_{1,2}$ were defined in eqs. $(3.25,3.26)$ of ref. [18],

$$
\mathcal{I}_{1}(\tau) \equiv \oiint_{K, Q} \frac{e^{i k_{n} \tau}}{Q^{2}(K-Q)^{2}}, \quad \mathcal{I}_{2}(\tau) \equiv £_{K, Q} \frac{k^{2} e^{i k_{n} \tau}}{K^{2} Q^{2}(K-Q)^{2}} .
$$

Graph ( $\mathrm{j}$ ) is a fairly complicated one, yielding

$$
\begin{aligned}
\delta_{(\mathrm{j})} G_{B}(\tau)= & \frac{g^{4} N_{\mathrm{c}} C_{\mathrm{F}}(D-3)(D-2)}{6} \mathcal{I}_{6}(\tau), \\
\mathcal{I}_{6}(\tau) \equiv & {\left[\int_{\tau}^{\beta} \mathrm{d} \tau^{\prime}-\int_{0}^{\tau} \mathrm{d} \tau^{\prime}\right] \int_{X} G\left(x_{0}-\tau^{\prime}, \mathbf{x}\right) } \\
& \times\left[\partial_{i} G\left(x_{0}-\tau, \mathbf{x}\right) \partial_{0} \partial_{i} G\left(x_{0}, \mathbf{x}\right)-\partial_{0} \partial_{i} G\left(x_{0}-\tau, \mathbf{x}\right) \partial_{i} G\left(x_{0}, \mathbf{x}\right)\right],
\end{aligned}
$$

where $X \equiv\left(x_{0}, \mathbf{x}\right)$. The function $\mathcal{I}_{6}$ is similar but not identical to $\mathcal{I}_{5}$, defined in eq. (3.18) of ref. [18]; in fact we get $\mathcal{I}_{6}$ if we set $D \rightarrow 3$ in a prefactor appearing in $\mathcal{I}_{5}$, and carry out a partial integration. Finally, the self-energy graph $(\mathrm{k})$ yields

$$
\begin{array}{r}
\delta_{(\mathrm{k})} G_{B}(\tau)=\frac{g^{4} C_{\mathrm{F}}(D-3)}{3}\left\{(D-2) N_{\mathrm{c}}\left[-\frac{(D-2)(D-1)}{2} \mathcal{I}_{0}(\tau)+2 \mathcal{I}_{2}(\tau)+2 \mathcal{I}_{7}(\tau)\right]\right. \\
\left.+N_{\mathrm{f}}\left[(D-2)(D-1) \mathcal{I}_{\{0\}}(\tau)-(D-2) \mathcal{I}_{\{2\}}(\tau)-4 \mathcal{I}_{\{7\}}(\tau)\right]\right\},
\end{array}
$$

where the four-momentum $Q$ is fermionic in $\mathcal{I}_{\{0\}}, \mathcal{I}_{\{2\}}$ and $\mathcal{I}_{\{7\}}$; we have made use of the identity

$$
\int_{\mathbf{k}} \frac{k^{2}}{K^{4}}=\frac{D-1}{2} \int_{\mathbf{k}} \frac{1}{K^{2}}
$$

in order to express results in terms of the factorizable structure $\mathcal{I}_{0}$ defined as

$$
\mathcal{I}_{0}(\tau) \equiv \oiint_{K, Q} \frac{e^{i k_{n} \tau}}{K^{2}(K-Q)^{2}}
$$


and we have denoted

$$
\mathcal{I}_{7}(\tau) \equiv-\lim _{\lambda \rightarrow 0} \frac{\mathrm{d}}{\mathrm{d} \lambda^{2}} \oiint_{K, Q} \frac{\left[k^{2} q^{2}-(\mathbf{k} \cdot \mathbf{q})^{2}\right] e^{i k_{n} \tau}}{\left(K^{2}+\lambda^{2}\right) Q^{2}(K-Q)^{2}} .
$$

Even if not obvious at first sight, the thermal part of $\mathcal{I}_{7}$ turns out to be closely related to the thermal part of $\mathcal{I}_{3}$, defined in eq. (3.27) of ref. [18].

In order to extract $\kappa_{B}$, we denote, in accordance with eqs. (4.4)-(4.6),

$$
\left.\tilde{\mathcal{I}}_{i}(\omega) \equiv \operatorname{Im} \int_{0}^{\beta} \mathrm{d} \tau e^{i \omega_{n} \tau} \mathcal{I}_{i}(\tau)\right|_{\omega_{n} \rightarrow-i\left[\omega+i 0^{+}\right]}, \quad \kappa_{i} \equiv \lim _{\omega \rightarrow 0} \frac{2 T \tilde{\mathcal{I}}_{i}(\omega)}{\omega} .
$$

The functions $\tilde{I}_{1,2,4}(\omega)$ are given in eqs. (A.54,55,57), respectively, of ref. [18], whereas $\tilde{I}_{0}$ can be extracted from eq. (B.9), multiplied by the tadpole $\mathbb{E}_{Q} \frac{1}{Q^{2}}=\frac{T^{2}}{12}$ or $\mathbb{\&}_{\{Q\}} \frac{1}{Q^{2}}=-\frac{T^{2}}{24}$. This leads to

$$
\kappa_{0}=\frac{T^{3}}{24 \pi}, \quad \kappa_{1}=\kappa_{2}=\frac{T^{3}}{12 \pi}, \quad \kappa_{4}=-\frac{T^{3}}{12 \pi}, \quad \kappa_{\{0\}}=-\frac{T^{3}}{48 \pi}, \quad \kappa_{\{2\}}=-\frac{T^{3}}{24 \pi} .
$$

For the function $\tilde{\mathcal{I}}_{6}$, originating from eq. (4.18), we find the thermal part

$$
\begin{aligned}
\tilde{\mathcal{I}}_{6}(\omega) \supset \frac{1}{8 \pi^{3}} \int_{0}^{\infty} \mathrm{d} q n_{\mathrm{B}}(q) \mathbb{P}\{ & 4 q \omega\left(1+\frac{\omega^{2}}{\omega^{2}-q^{2}}\right)+5 \omega^{2} \ln \left|\frac{q+\omega}{q-\omega}\right| \\
& \left.+\frac{2 \omega^{4}}{q}\left[\frac{1}{q+\omega} \ln \frac{q+\omega}{\omega}-\frac{1}{q-\omega} \ln \frac{|q-\omega|}{\omega}\right]\right\},
\end{aligned}
$$

where $\mathbb{P}$ denotes a principal value. The small- $\omega$ limit amounts to

$$
\kappa_{6}=\frac{T^{3}}{6 \pi} .
$$

The function $\tilde{\mathcal{I}}_{7}(\omega)$, originating from eq. (4.22), can be obtained in close analogy with the discussion in appendices A.1-3 of ref. [18], and its thermal part agrees, up to a sign, with that of $\tilde{\mathcal{I}}_{3}(\omega)$ in eq. (A.56) of ref. [18]:

$$
\tilde{\mathcal{I}}_{7}(\omega) \supset \frac{1}{16 \pi^{3}} \int_{0}^{\infty} \mathrm{d} q n_{\mathrm{B}}(q)\left\{q^{2} \ln \left|\frac{q+\omega}{q-\omega}\right|+q \omega \ln \left|\frac{q^{2}-\omega^{2}}{\omega^{2}}\right|\right\} .
$$

In the fermionic $\tilde{\mathcal{I}}_{\{7\}}$, we need to replace $n_{\mathrm{B}} \rightarrow-n_{\mathrm{F}}$. The corresponding transport coefficients amount to

$$
\begin{gathered}
\kappa_{7} \approx \frac{T^{3}}{24 \pi}\left[\ln \left(\frac{T}{\omega}\right)+2-\gamma_{\mathrm{E}}+\frac{\zeta^{\prime}(2)}{\zeta(2)}\right], \\
\kappa_{\{7\}} \approx-\frac{T^{3}}{48 \pi}\left[\ln \left(\frac{2 T}{\omega}\right)+2-\gamma_{\mathrm{E}}+\frac{\zeta^{\prime}(2)}{\zeta(2)}\right],
\end{gathered}
$$

where " $\approx$ " indicates that the limit $\omega \rightarrow 0$ has not been taken inside the logarithm.

Summing up the effects from all the diagrams, we obtain

$$
\begin{aligned}
\kappa_{B}^{\mathrm{QCD}, \text { expanded }} \approx \frac{g^{4} C_{\mathrm{F}} T^{3}}{18 \pi}\{ & N_{\mathrm{c}}\left[\ln \left(\frac{T}{\omega}\right)+1-\gamma_{\mathrm{E}}+\frac{\zeta^{\prime}(2)}{\zeta(2)}\right] \\
& \left.+\frac{N_{\mathrm{f}}}{2}\left[\ln \left(\frac{2 T}{\omega}\right)+\frac{3}{2}-\gamma_{\mathrm{E}}+\frac{\zeta^{\prime}(2)}{\zeta(2)}\right]\right\} .
\end{aligned}
$$

Clearly the result is IR-divergent for $\omega \rightarrow 0$, and necessitates resummation. 


\subsubsection{Hard Thermal Loop resummation}

Plasma effects from the colour-electric scale, $m_{\mathrm{E}}^{2} \stackrel{D \equiv}{=} g^{2} T^{2}\left(\frac{N_{\mathrm{c}}}{3}+\frac{N_{\mathrm{f}}}{6}\right)$, can be incorporated by carrying out HTL resummation [19-22]. The ingredients needed for the current problem are described in appendix B of ref. [18], and can be adapted to $G_{B}$ with minor modifications. For the "expanded" version we obtain, in analogy with eq. (B.14) of ref. [18],

$$
\kappa_{B}^{\mathrm{HTL}, \text { expanded }} \approx \frac{2 g^{2} C_{\mathrm{F}} m_{\mathrm{E}}^{2} T(D-3)}{3} \frac{\mu^{-2 \epsilon}}{8 \pi}\left(\frac{1}{\epsilon}+\ln \frac{\bar{\mu}^{2}}{4 \omega^{2}}-1\right) .
$$

The "full" HTL computation does not regularize $G_{B}$, as colour-magnetic fields play a role. We introduce an intermediate $a d$ hoc IR regulator, $m_{\mathrm{G}}$, as a colour-magnetic scale, and represent the full HTL result, in analogy with eq. (B.18) of ref. [18], as

$$
\begin{aligned}
\kappa_{B}^{\mathrm{HTL}, \mathrm{full}} & =\frac{2 g^{2} C_{\mathrm{F}} m_{\mathrm{E}}^{2} T(D-3)}{3} \lim _{m_{\mathrm{G}}^{2} \rightarrow 0} \int_{\mathbf{k}} \frac{\mathbf{k}^{2} \pi \delta(\mathbf{k} \cdot \mathbf{v})}{\left(\mathbf{k}^{2}+m_{\mathrm{G}}^{2}\right)^{2}} \\
& =\frac{2 g^{2} C_{\mathrm{F}} m_{\mathrm{E}}^{2} T(D-3)}{3} \lim _{m_{\mathrm{G}}^{2} \rightarrow 0} \frac{\mu^{-2 \epsilon}}{8 \pi}\left(\frac{1}{\epsilon}+\ln \frac{\bar{\mu}^{2}}{m_{\mathrm{G}}^{2}}-1\right) .
\end{aligned}
$$

Clearly the result is IR divergent; it is rendered finite by non-perturbative dynamics at the colour-magnetic scale $\sim \alpha_{s} N_{\mathrm{c}} T$ [23]. The difference $\kappa_{B}^{\mathrm{HTL} \text {,full }}-\kappa_{B}^{\text {HTL,expanded }}$, appearing in eq. (4.7), is however UV finite as it should (i.e. $1 / \epsilon$ cancels), and effectively replaces the $\ln \omega$ 's in eq. (4.30) with logarithms of the colour-magnetic scale.

\subsubsection{Summary}

Summing up the effects from eqs. (4.30), (4.31), (4.32) according to eq. (4.7), and replacing the artificial scale $m_{\mathrm{G}}$ by the physical colour-magnetic scale $\alpha_{\mathrm{S}} N_{\mathrm{c}} T$, which however is associated with a process-dependent non-perturbative constant, $c_{B}$, we obtain

$$
\begin{aligned}
\kappa_{B}=\frac{g^{4} C_{\mathrm{F}} T^{3}}{18 \pi}\{ & N_{\mathrm{c}}\left[\ln \left(\frac{2 c_{B}}{\alpha_{s} N_{\mathrm{c}}}\right)+1-\gamma_{\mathrm{E}}+\frac{\zeta^{\prime}(2)}{\zeta(2)}\right] \\
& \left.+\frac{N_{\mathrm{f}}}{2}\left[\ln \left(\frac{4 c_{B}}{\alpha_{s} N_{\mathrm{c}}}\right)+\frac{3}{2}-\gamma_{\mathrm{E}}+\frac{\zeta^{\prime}(2)}{\zeta(2)}\right]\right\}+\mathcal{O}\left(g^{5}\right) .
\end{aligned}
$$

Even if $c_{B}$ remains unknown, the coefficients of the logarithms are unambiguously predicted, as they characterize contributions from a ratio of scales. It is worth remarking that $1-$ $\gamma_{\mathrm{E}}+\frac{\zeta^{\prime}(2)}{\zeta(2)} \approx-0.147$, so the argument of the first logarithm should exceed 1.16 for a sensible result.

The expression for $\kappa_{B}$ is quite similar to that for $\kappa_{E}$ [14], with the difference that in the latter IR sensitivity is regularized by $m_{\mathrm{E}}$ rather than $\alpha_{\mathrm{S}} N_{\mathrm{c}} T$. In the extreme weak-coupling limit, $\alpha_{\mathrm{S}} N_{\mathrm{c}} T \ll m_{\mathrm{E}}$, so we might expect $\kappa_{B}$ to be larger than $\kappa_{E}$, however the difference is inside a logarithm so this argument is weak. In general, colour-magnetic corrections tend to be large compared with those from the scale $m_{\mathrm{E}}$ (cf., e.g., ref. [24] for a review), however NLO corrections to $\kappa_{E}$ are large and positive [15], so it is hard to anticipate whether there is a clear hierarchy between $\kappa_{E}$ and $\kappa_{B}$ at practically reachable temperatures. 


\section{Conclusions and outlook}

The purpose of the present paper has been to consider effects of relative order $\mathcal{O}(T / M)$ to the heavy quark momentum diffusion coefficient, $\kappa$. We have argued that one correction originates from a colour-magnetic correlator dressing a Polyakov loop (cf. eq. (3.39)), and that the corresponding contribution to $\kappa$, denoted by $\kappa_{B}$ (cf. eq. (3.40)), is non-perturbative already at leading order of the weak-coupling expansion (cf. eq. (4.33)). Apart from the known dramatic increase of $\kappa_{E}$ through interactions [8-13, 15], additional $\mathcal{O}(T / M)$-effects originating from $\kappa_{B}$ could help to explain why charm quarks show fast kinetic equilibration at temperatures not much above the confinement scale (cf., e.g., ref. [1]).

It should be stressed, however, that $\kappa_{B}$ does not represent the only potential $\mathcal{O}(T / M)$ effect. On the side of rate coefficients, further corrections of $\mathcal{O}(T / M)$ could be eliminated by taking care to avoid secular terms (cf. point (ii) in section 2), by using covariant momenta in Langevin simulations (cf. point (iii) in section 2), and by including corrections in the relation of $\kappa$ and $\eta$ (cf. point (iv) in section 2). On the side of dispersive effects, a correction originates from the difference between the vacuum pole mass $M$ and the thermal kinetic mass $M_{\text {kin }}$, even if it is difficult to quantify this effect, given that $M$ is ambiguous by $\sim \mathcal{O}\left(\Lambda_{\overline{\mathrm{MS}}}\right)$; in practice, $M_{\text {kin }}$ should probably be treated as a fit parameter. In spite of these additional ingredients, we hope that an estimate of $\kappa_{B}$ could give a fair impression about the size of finite-mass corrections to heavy quark rate observables.

As far as a lattice study of $\kappa_{B}$ goes, the prospects look quite good. Previous investigations of the colour-electric correlator [8-13] show that a signal can be obtained, and a continuum extrapolation is feasible. On the aspect of renormalization, where only perturbative factors have been worked out for $\kappa_{E}$ [25], the non-perturbative level has been reached for a particular discretization of colour-magnetic fields [26]. Conceivably, gradient flow [27] could offer further tools for studying the colour-magnetic correlator, along the lines discussed in ref. [28], and physical insight could be obtained from classical lattice gauge theory simulations $[29,30]$. Finally, it might be worth considering whether the colour-magnetic correlator captures some dispersive effects as well, in analogy with the colour-electric one [31].

\section{Acknowledgments}

M.L. thanks Debasish Banerjee for suggesting the topic many years ago and for his continued interest in it, and Saumen Datta for helpful discussions. Our work was partly supported by the Swiss National Science Foundation (SNF) under grant 200020B-188712.

Open Access. This article is distributed under the terms of the Creative Commons Attribution License (CC-BY 4.0), which permits any use, distribution and reproduction in any medium, provided the original author(s) and source are credited. 


\section{References}

[1] R. Rapp et al., Extraction of heavy-flavor transport coefficients in QCD matter, Nucl. Phys. A 979 (2018) 21 [arXiv:1803.03824] [InSPIRE].

[2] J.F. Donoghue, B.R. Holstein and R.W. Robinett, Quantum electrodynamics at finite temperature, Annals Phys. 164 (1985) 233 [Erratum ibid. 172 (1986) 483] [INSPIRE].

[3] L.S. Brown and R.F. Sawyer, Nuclear reaction rates in a plasma, Rev. Mod. Phys. 69 (1997) 411 [astro-ph/9610256] [INSPIRE].

[4] P.M. Chesler, A. Gynther and A. Vuorinen, On the dispersion of fundamental particles in $Q C D$ and $\mathcal{N}=4$ super Yang-Mills theory, JHEP 09 (2009) 003 [arXiv:0906.3052] [INSPIRE].

[5] P. Petreczky and D. Teaney, Heavy quark diffusion from the lattice, Phys. Rev. D 73 (2006) 014508 [hep-ph/0507318] [InSPIRE].

[6] J. Casalderrey-Solana and D. Teaney, Heavy quark diffusion in strongly coupled $\mathcal{N}=4$ Yang-Mills, Phys. Rev. D 74 (2006) 085012 [hep-ph/0605199] [InSPIRE].

[7] S. Caron-Huot, M. Laine and G.D. Moore, A way to estimate the heavy quark thermalization rate from the lattice, JHEP 04 (2009) 053 [arXiv:0901.1195] [INSPIRE].

[8] H.B. Meyer, The errant life of a heavy quark in the quark-gluon plasma, New J. Phys. 13 (2011) 035008 [arXiv: 1012.0234] [INSPIRE].

[9] A. Francis, O. Kaczmarek, M. Laine and J. Langelage, Towards a non-perturbative measurement of the heavy quark momentum diffusion coefficient, PoS (LATTICE2011) 202 (2011) [arXiv:1109.3941] [INSPIRE].

[10] D. Banerjee, S. Datta, R. Gavai and P. Majumdar, Heavy quark momentum diffusion coefficient from lattice QCD, Phys. Rev. D 85 (2012) 014510 [arXiv:1109.5738] [INSPIRE].

[11] A. Francis, O. Kaczmarek, M. Laine, T. Neuhaus and H. Ohno, Nonperturbative estimate of the heavy quark momentum diffusion coefficient, Phys. Rev. D 92 (2015) 116003 [arXiv: 1508.04543] [INSPIRE].

[12] N. Brambilla, V. Leino, P. Petreczky and A. Vairo, Lattice QCD constraints on the heavy quark diffusion coefficient, Phys. Rev. D 102 (2020) 074503 [arXiv:2007.10078] [InSPIRE].

[13] L. Altenkort, A.M. Eller, O. Kaczmarek, L. Mazur, G.D. Moore and H.-T. Shu, Heavy quark momentum diffusion from the lattice using gradient flow, arXiv:2009.13553 [INSPIRE].

[14] G.D. Moore and D. Teaney, How much do heavy quarks thermalize in a heavy ion collision?, Phys. Rev. C 71 (2005) 064904 [hep-ph/0412346] [INSPIRE].

[15] S. Caron-Huot and G.D. Moore, Heavy quark diffusion in $Q C D$ and $\mathcal{N}=4 S Y M$ at next-to-leading order, JHEP 02 (2008) 081 [arXiv:0801.2173] [INSPIRE].

[16] Y. Burnier and M. Laine, Massive vector current correlator in thermal QCD, JHEP 11 (2012) 086 [arXiv:1210.1064] [INSPIRE].

[17] A.V. Manohar, Heavy quark effective theory and nonrelativistic QCD Lagrangian to order $\alpha_{s} / m^{3}$, Phys. Rev. D 56 (1997) 230 [hep-ph/9701294] [INSPIRE].

[18] Y. Burnier, M. Laine, J. Langelage and L. Mether, Colour-electric spectral function at next-to-leading order, JHEP 08 (2010) 094 [arXiv: 1006.0867] [INSPIRE]. 
[19] R.D. Pisarski, Scattering amplitudes in hot gauge theories, Phys. Rev. Lett. 63 (1989) 1129 [INSPIRE].

[20] J. Frenkel and J.C. Taylor, High temperature limit of thermal QCD, Nucl. Phys. B 334 (1990) 199 [InSPIRE].

[21] E. Braaten and R.D. Pisarski, Soft amplitudes in hot gauge theories: a general analysis, Nucl. Phys. B 337 (1990) 569 [InSPIRE].

[22] J.C. Taylor and S.M.H. Wong, The effective action of hard thermal loops in QCD, Nucl. Phys. B 346 (1990) 115 [INSPIRE].

[23] A.D. Linde, Infrared problem in thermodynamics of the Yang-Mills gas, Phys. Lett. B 96 (1980) 289 [INSPIRE].

[24] M. Laine and M. Vepsäläinen, On the smallest screening masses in hot QCD, JHEP 09 (2009) 023 [arXiv:0906.4450] [INSPIRE].

[25] C. Christensen and M. Laine, Perturbative renormalization of the electric field correlator, Phys. Lett. B 755 (2016) 316 [arXiv:1601.01573] [INSPIRE].

[26] D. Guazzini, H.B. Meyer and R. Sommer, Non-perturbative renormalization of the chromo-magnetic operator in heavy quark effective theory and the $B^{*}-B$ mass splitting, JHEP 10 (2007) 081 [arXiv:0705.1809] [INSPIRE].

[27] M. Lüscher, Properties and uses of the Wilson flow in lattice QCD, JHEP 08 (2010) 071 [Erratum ibid. 03 (2014) 092] [arXiv:1006.4518] [INSPIRE].

[28] A.M. Eller and G.D. Moore, Gradient-flowed thermal correlators: how much flow is too much?, Phys. Rev. D 97 (2018) 114507 [arXiv: 1802.04562] [INSPIRE].

[29] M. Laine, G.D. Moore, O. Philipsen and M. Tassler, Heavy quark thermalization in classical lattice gauge theory: lessons for strongly-coupled QCD, JHEP 05 (2009) 014 [arXiv: 0902.2856] [INSPIRE].

[30] K. Boguslavski, A. Kurkela, T. Lappi and J. Peuron, Heavy quark diffusion in an overoccupied gluon plasma, JHEP 09 (2020) 077 [arXiv: 2005. 02418] [INSPIRE].

[31] A.M. Eller, J. Ghiglieri and G.D. Moore, Thermal heavy quark self-energy from Euclidean correlators, Phys. Rev. D 99 (2019) 094042 [Erratum ibid. 102 (2020) 039901] [arXiv: 1903.08064] [INSPIRE]. 